\title{
Multidisciplinary sarcoma tumor board: retroperitoneal liposarcoma
}

\author{
William W. Tseng ${ }^{1}$, Jun Chen ${ }^{2}$, Dakshesh Patel ${ }^{1}$, Chengli Miao' ${ }^{2}$ Alisa Ching ${ }^{1}$, Shaomin Yang ${ }^{3}$, \\ Yuanchun Feng ${ }^{2}$, George Matcuk ${ }^{1}$, Kristi Lara ${ }^{1}$, James Hu ${ }^{1}$, Omar Ragab ${ }^{1}$, Jennifer Ho ${ }^{1}$, Xiaosong Rao ${ }^{2}$, \\ Shahbaz Hanif ${ }^{2,4}$, Chenghua Luo ${ }^{2,4}$
}

${ }^{1}$ Departments of Surgery, Radiology, Medical Oncology, and Radiation Oncology, Keck School of Medicine, University of Southern California (USC) and Norris Comprehensive Cancer Center, Los Angeles, California, USA; ${ }^{2}$ Departments of Surgery, Radiology, and Pathology, Peking University International Hospital (PKUIH), Beijing 102206, China; ${ }^{3}$ Department of Pathology, Peking University Third Hospital, Beijing 100083, China; ${ }^{4}$ Beijing Spanal Medical Scientific Co. Ltd., Beijing 102206, China

Correspondence to: William W. Tseng, MD. USC Surgical Oncology, 1510 San Pablo Street, HCC-I, Suite 514, Los Angeles, CA 90033, USA. Email: william.tseng@med.usc.edu; Chenghua Luo, MD, PhD. Department of Retroperitoneal Tumor Surgery, Peking University International Hospital, Life Park Road No. 1 Life Science Park of Zhong Guancun, Chang Ping District, Beijing 102206, China. Email: luochenghua@pkuih.edu.cn.

Submitted Dec 28, 2019. Accepted for publication Feb 12, 2020.

doi: $10.21037 /$ cco.2020.02.08

View this article at: http://dx.doi.org/10.21037/cco.2020.02.08

Liposarcoma is a malignancy of fat that commonly develops in the retroperitoneum $(1,2)$. In this anatomic location, tumors can be low grade, well differentiated (WD) or have an additional high grade, dedifferentiated (DD) component. Entirely WD tumors are typically indolent and do not metastasize, while tumors with a DD component are more aggressive and have distant metastatic potential. WD and DD liposarcoma can be regarded as opposite ends of a spectrum of disease aggressiveness (e.g., lipoma-like WD to rhabdomyoblastic DD) (3).

For patients with retroperitoneal (RP) liposarcoma, high resolution, contrast enhanced CT of the abdomen and pelvis is critical as part of the initial evaluation (4). This should be performed if not done already at the time of referral. WD liposarcomas are well-defined round or lobulated masses predominantly composed of macroscopic fat with few, thin septations (5). Non-fatty, soft tissue density areas with a ground glass appearance can occur and typically constitute less than $25 \%$ of the tumor (6). DD areas manifest as discrete nodular soft tissue density areas often with good demarcation from surrounding fat and show variable contrast enhancement. Internal septations are thicker $(>2 \mathrm{~mm})$ in DD. Calcifications may also be seen and would suggest DD liposarcoma if found in the nonfatty areas. For patients with suspected DD disease, CT of the chest should be obtained as part of staging to rule out distant metastasis to the lungs (4).

To confirm the diagnosis of RP liposarcoma, percutaneous core needle biopsy can be performed. Biopsy may not always be necessary, especially in cases in which the radiologic features highly suggest the diagnosis. On histopathologic examination, WD liposarcoma consists of adipocytes of varying size with fibrous septae and interspersed hyperchromatic cells (7). DD areas are typically juxtaposed next to WD areas with an abrupt transition from the adipocytic (WD) to non-adipocytic, high grade appearing areas containing prominent mitotic figures (DD). Both WD and DD liposarcoma have amplification of 12q13-15 which includes several important genes such as MDM2 and CDK4. This molecular feature is considered pathognomonic and can be used for the definitive diagnosis if the histologic features alone are unclear.

For all patients with nonmetastatic retroperitoneal liposarcoma, surgery is the mainstay of treatment. However, in the retroperitoneum, tumors are frequently massive in size and can potentially invade critical organs and major blood vessels, making surgery very difficult. Whether due to technical factors (e.g., "positive" margins) or disease biology [e.g., "field defect" (8)], recurrence rates after resection are high and do not plateau over time (9). Radiation therapy may offer improved local control, but the true benefit for retroperitoneal liposarcoma remains controversial (10). 

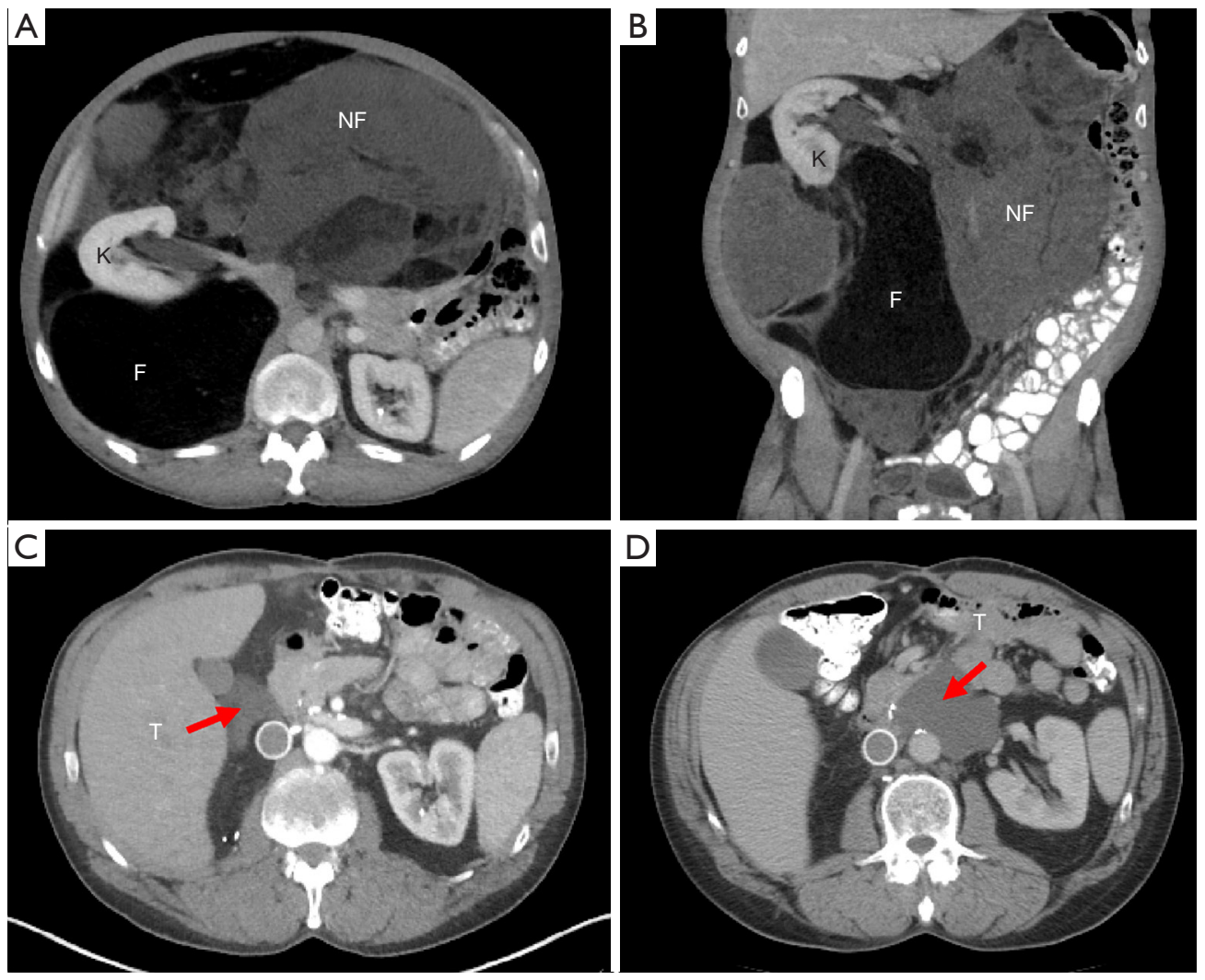

Figure 1 Axial post contrast (A, at the level of the kidneys) and coronal reformat (B) CT images of a large heterogeneous tumor originating on the right side and occupying most of the abdomen. The mass has fatty (F) and non-fatty (NF) components and encircles and displaces the right kidney $(\mathrm{K})$. The patient developed first recurrence $(\mathrm{C})$ with a tumor $(\mathrm{T}$, red arrow) in the right upper abdomen between the gall bladder, duodenum and inferior vena cava graft. The patient later developed second recurrence (D) with a tumor (T, red arrow) anterior to the aorta.

Many systemic therapy options are available, including cytotoxic chemotherapy, targeted agents, marine-derived drugs and immunotherapy. ; however tumor response rates are overall low (11).

For this multidisciplinary sarcoma tumor board, our institutions discussed two cases of retroperitoneal liposarcoma to highlight some of the relevant clinical issues and challenges with this disease.

\section{Case 1 (USC)}

A 58-year-old male, otherwise healthy, developed progressive abdominal fullness and bilateral inguinal hernias over the course of a year. After hernia repair, a CT scan was done which identified a giant soft tissue mass occupying the majority of the abdomen (Figure 1). The tumor had both lipomatous and non-lipomatous components with marked displacement of the abdominal viscera to the left. The entire right kidney and the inferior vena cava (IVC) just below the level of the renal vessels were surrounded by the tumor. Percutaneous core needle biopsy was obtained pre-referral and found to be consistent with low grade (WD) liposarcoma. The decision was made to proceed with surgery. Complete resection was achieved including en bloc resection of the right kidney, right colon and infrarenal IVC with inline graft reconstruction. The tumor measured approximately $50 \mathrm{~cm}$ and weighed $15 \mathrm{~kg}$ (Figure $2 A, B$ ). Final pathology was consistent with WD disease with increased cellularity but without a clear high grade (DD) component. Incidentally, the resected right kidney harbored a $3 \mathrm{~cm}$ angiomyolipoma and a small nodule excised from the anterior stomach was consistent with gastrointestinal stromal tumor.

The patient was closely followed in surveillance with 

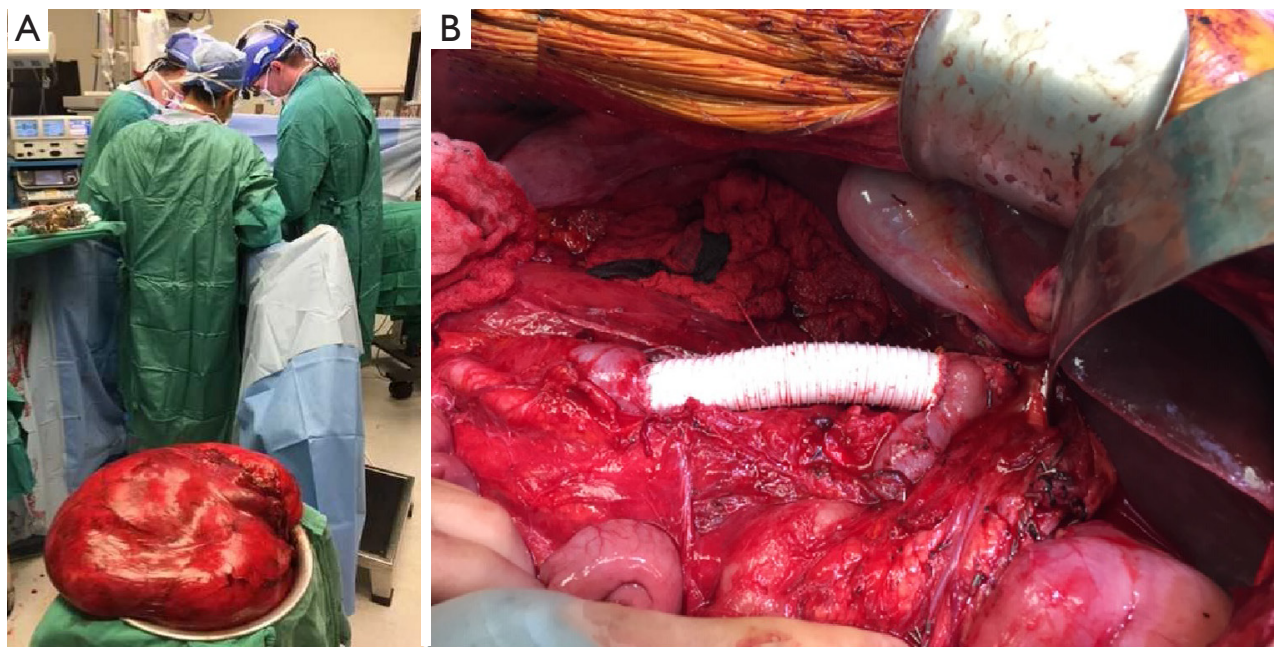

Figure 2 Photograph of the resected tumor specimen (A) and right retroperitoneum after resection (B) with inferior vena cava graft (white).

serial CT scans. Extensive postoperative changes were noted. However, at 10 months from surgery, there was definitive evidence of local recurrence at the liver hilum (Figure 1C). The patient strongly preferred surgery rather than other upfront therapies. At this surgery, complete resection was achieved and no other sites of obvious disease were noted. Final pathology for the tumor again showed WD disease without DD. At the follow-up CT scan only 4 months later, there appeared to be a soft tissue mass on the contralateral left side, anterior to the aorta at the level of the pancreas neck/body. Lymphoma or other cancer (given his history) was in the differential diagnosis and endoscopic biopsy was done but was nondiagnostic. The decision was made to initially observe this mass, however subsequent CT demonstrated clear growth in size (Figure 1D), potentially threatening the hilar vessels for his solitary left kidney. The patient again declined nonsurgical therapies and underwent his third surgery. The tumor was completely resected while preserving the pancreas, aorta and left renal artery and vein. Again, no other sites of disease were noted at surgery. Final pathology was consistent with WD liposarcoma without DD. The patient declined adjuvant therapy and is currently being followed in surveillance.

\section{Radiologic discussion}

Surveillance is important after surgery for retroperitoneal liposarcoma. While there are no specific guidelines, we would agree with most other sarcoma centers that high resolution CT of the abdomen/pelvis with contrast should be done every 4 to 6 months for the first 2 to 3 years, followed by 6 months to annually for at least to 5 years (12). CT of the chest should be included for patients with DD tumors. Importantly, we recommend a postoperative "baseline" CT to establish lack of residual disease (e.g., first CT at 4 months). One issue highlighted in this case is that particularly for large tumors and complex resections, there can be initial ambiguity in distinguishing postoperative changes from true recurrence. This may be resolved by needle biopsy or simply observation for continued growth suggestive of tumor on serial CT scans. In some situations, especially for WD disease, known recurrences can continue to be observed by serial CT scans without treatment $(13,14)$. This is to study the disease behavior, assessing tumor growth rate and monitoring for development of multifocal sites of disease. In some patients, as in this case, local recurrences may even develop in areas which seem remote from the original resection site (8). The timing of surgical intervention for recurrent tumors should be discussed in a multidisciplinary setting, incorporating disease behavior, clinical factors such as patient symptoms, and impending threat to organs and critical structures.

\section{Pathologic discussion}

None specific to this patient's retroperitoneal liposarcoma. Interestingly, this patient had a synchronous second primary cancer (e.g., gastrointestinal stromal tumor). We have previously reported that some patients with liposarcoma seem to have a propensity to develop this without a clearly 
defined hereditary cancer syndrome (15). We hypothesize that in these patients, there may be an underlying genetic abnormality or possibly a defect in systemic immune surveillance, particularly when such large liposarcoma tumors are intact.

\section{Treatment discussion}

The surgical oncologist felt that complete resection (R0/R1) of all gross disease was achieved, yet definitive radiologic evidence of recurrence developed fairly rapidly in this patient. At the time of initial surgery, organs (right kidney, colon and IVC) were removed en bloc with the tumor due to obvious involvement. For retroperitoneal liposarcoma, others have advocated extended or compartmental resection which is a more liberal policy of organ resection even without obvious involvement $(16,17)$. The goal is to minimize microscopically "positive" margins. The reported retrospective data suggests that this surgical approach does lower local recurrence rates. In this patient, for such a large tumor, the surgical oncologist felt that extended resection would have necessitated a Whipple procedure, with its associated morbidity. Hypothetically, even if this were done, it still would not have addressed the recurrence he ultimately developed on the opposite side. To try to surgically clear all potential sites of disease, the surgical oncologists at PKUIH in Beijing have described the concept of total ipsilateral retroperitoneal lipectomy (TRL). In this specific case, however, the tumor would have required bilateral TRL which is not feasible.

With regard to nonsurgical treatments, our radiation oncologists felt that the primary tumor in this patient was too large to radiate from a technical standpoint. Radiation therapy would have been more feasible and could have been considered for the recurrent tumors, which were smaller. Our surgical oncologist expressed hesitation about the potential for radiation therapy to make subsequent surgery more challenging due to scar tissue, particularly around major vessels such as the left renal vein for the patient's solitary kidney at the third resection. We also discussed the various options for systemic therapy in retroperitoneal liposarcoma that could have been considered if this patient had been willing to undergo nonsurgical treatment. Importantly, however, there is currently a paucity of effective systemic therapies for WD disease $(11,14)$. At initial presentation and with each of his subsequent recurrences, his tumors never had evidence of DD disease, which limits systemic therapy options. Further research is clearly needed to provide better treatment to patients with retroperitoneal liposarcoma.

\section{Case 2 (PKUIH)}

A 64-year-old female was found to have a $14 \mathrm{~cm} \mathrm{left}$ perinephric mass and underwent surgery with en bloc nephrectomy by her local physicians. Final pathology showed WD liposarcoma with lipoma-like component. On surveillance imaging approximately a year and a half after surgery, the patient was found to have a $9 \mathrm{~cm}$ lesion in the contralateral right retroperitoneum causing hydronephrosis. Percutaneous core needle biopsy confirmed this was high grade (DD) recurrence. It was felt that upfront systemic therapy was best, and the patient received a regimen consisting of anlotinib and epirubicin. However, she did not finish the intended treatment course due to severe toxicity including nausea and vomiting. She was then referred to our center for surgical evaluation for her recurrent disease.

Multidisciplinary review of her case was done prior to surgery. Staging work-up did not demonstrate any obvious distant metastasis. Interval CT now showed that the recurrent tumor was much larger, in fact occupying almost the entirety of the abdomen and retroperitoneum. Importantly, the whole length of infrahepatic IVC appeared to be involved, although the bilateral iliac veins remained patent (Figure 3A). The duodenum, pancreatic head, and right renal vein/ureter to her solitary kidney were all displaced and compressed. There was no evidence of bony invasion to the spine or pelvis.

Complete resection (R0/R1) of the tumor was achieved by our surgical team (Figure 3B,C). The IVC was injured and repaired, and the right ureter was transected requiring reconstruction. However, the duodenum, pancreas and right kidney were preserved. The tumor measured $38 \mathrm{~cm}$ and weighed $6.4 \mathrm{~kg}$. Final pathology was consistent with DD liposarcoma with a myxofibrosarcomatous component. Based on the FNCLCC grading system, the tumor was given a grade 3 out of 3 . The patient declined further adjuvant systemic therapy. No signs of local recurrence or disease metastasis were noted on surveillance CT scan, 9 months after surgery.

\section{Radiologic discussion}

None specific to this patient's retroperitoneal liposarcoma. We reviewed the imaging together and discussed technical considerations such as potential need for resection of the 

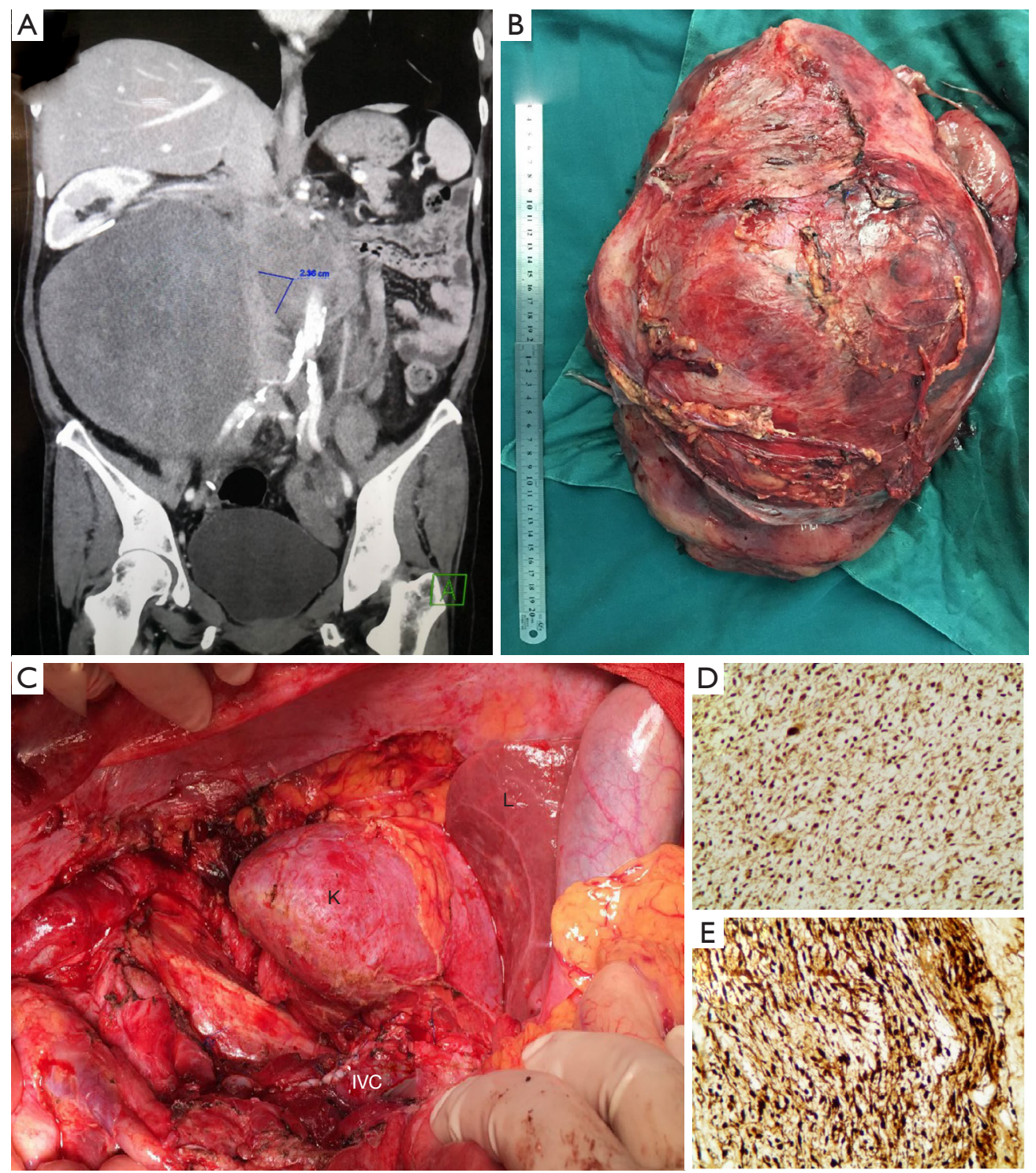

Figure 3 Coronal reformat (A) CT image of a large tumor that appears to involve the inferior vena cava (IVC). The mass was resected completely $(\mathrm{B}, \mathrm{C})$ while preserving the IVC and solitary right kidney (K; liver, L). By immunohistochemistry, the tumor expresses MDM2 (D) and CDK4 (E).

major vessels (e.g., IVC), duodenum, and head of pancreas while preserving her solitary right kidney. Importantly, it was pointed out in mutual agreement that although CT can help the surgeon anticipate resection versus preservation of tumor-adjacent organs and structures, ultimately these decisions are made intraoperatively.

\section{Pathologic discussion}

This case presented to our center at the time of recurrence.
Histological examination revealed spindle cell sarcoma with moderate cellularity without a clear WD component. Necrosis was observed focally. Overexpression of MDM2 and CDK4 was identified by immunohistochemistry (IHC) (Figure 3D,E). In this situation, the combination of clinical history and histologic features supported by IHC, our pathologist felt that molecular testing by fluorescence in situ hybridization (FISH) was not necessary. This can still be a valuable diagnostic tool in other cases when the diagnosis is not as clear $(18,19)$. In our discussion, we remarked that in 
this patient the progression from a primary, low grade WD tumor with lipoma-like features to recurrent high grade (FNCLCC 3/3) DD, highlighting the aggressive nature of the disease.

\section{Treatment discussion}

This was a very challenging surgical case, even for our experienced, high volume referral center. We attempted to preserve the right renal vein at all costs given that this was the patient's solitary kidney. Similarly, careful dissection was performed to preserve the duodenum and head of pancreas, avoiding Whipple procedure or pancreaticoduodenectomy. In a combined multiinstitutional series, pancreaticoduodenectomy combined with retroperitoneal sarcoma resection (for all subtypes combined) was associated with major complications in 34\% patients and postoperative death in $3.4 \%$ (20). From an oncologic standpoint, $66 \%$ of these patients still developed disease recurrence within five years. Consistent with this, performance of pancreaticoduodenectomy is independently associated with higher morbidity in retroperitoneal sarcoma surgery, based on additional data from the Trans-Atlantic Retroperitoneal Sarcoma Working Group (TARSWG) (21). At our center, pancreaticoduodenectomy is only indicated when there is clear evidence of tumor infiltration of pancreatic head, ampulla, or duodenum, or in patients with preoperative obstructive jaundice. With regards to the major vessels, although the IVC although the IVC initially appeared to be involved, with careful dissection, a plane was found separating this from the tumor and, therefore, this was preserved. Whether to resect or preserve the IVC is a continued area of controversy. Some have suggested that resection should be indicated if the vessel is surrounded by tumor for more than 180 degrees $(22,23)$. If resection is performed, a second issue is whether or not to reconstruct IVC. We have previously reported that reconstruction is not required if the patient has (I) a history of greater than a year with sufficient collateral circulation; (II) 75 percent or more obstruction of the IVC; and (III) a positive intraoperative furosemide injection test $(24,25)$. The latter consists of production of $100 \mathrm{~mL}$ of urine 30 minutes after temporary occlusion of the IVC and intravenous injection of $20 \mathrm{mg}$ furosemide.

Grade 3 DD liposarcoma of the retroperitoneum has a high risk of distant metastatic disease according to data from the TARPSWG (9). Given this risk, systemic therapy may have benefited this patient. Reported response rates for advanced and metastatic DD liposarcoma are 10-30\% by RECIST criteria $(26,27)$. The most commonly used chemotherapy regimens given in the front line setting include doxorubicin with or without ifosfamide versus gemcitabine/taxotere. Second line therapy such as eribulin or trabectedin could be considered on an individualized basis, however, data showing improved clinical outcomes (e.g., disease-free or overall survival) using any of these approaches in the setting of in the setting of resectable disease either as adjuvant or neoadjuvant therapy is lacking (11). Other systemic therapies include anti-PD-1 immunotherapy and targeted, CDK4 inhibitors are still under investigation $(28,29)$. Anlotinib, used in this patient, is a multikinase angiogenesis inhibitor, which has been shown to significantly prolong progression-free survival compared to placebo in patients with metastatic soft tissue sarcoma in a recent phase II study (30). Although not currently approved for standard use in the US or China for sarcoma, the data for anlotinib is promising and there is ongoing global investigation.

\section{Conclusions}

Retroperitoneal liposarcoma is a challenging malignancy with many important subtleties in diagnosis, treatment and surveillance. Presence of a high grade, DD component increases the aggressiveness of the disease, but even WD tumors can be difficult to manage. Although surgery is the mainstay of treatment, multidisciplinary discussion is important to consider the potential benefit of nonsurgical therapies (e.g., radiation, systemic therapy). We encourage global collaboration to learn from each other and streamline research efforts to ultimately find better treatment for this disease.

\section{Acknowledgments}

We would like to acknowledge Ms. Shu Li and Ms. Mei Huang, RN for their gracious assistance with audiovisual set-up to make these teleconferences possible.

Funding: None.

\section{Footnote}

Provenance and Peer Review: This article was commissioned by the Guest Editor (William W. Tseng) for the series "Sarcoma MDT". The article was sent for external peer review organized by the Guest Editor and the editorial 
office.

Conflicts of Interest: All authors have completed the ICMJE uniform disclosure form (available at http://dx.doi. org/10.21037/cco.2020.02.08). The series "Sarcoma MDT" was commissioned by the editorial office without any funding or sponsorship. WWT serves as an unpaid editorial board member of Chinese Clinical Oncology from Oct 2018 to Sep 2020 and served as the unpaid Guest Editor for the series. The other authors have no conflicts of interest to declare.

Ethical Statement: The authors are accountable for all aspects of the work in ensuring that questions related to the accuracy or integrity of any part of the work are appropriately investigated and resolved.

Open Access Statement: This is an Open Access article distributed in accordance with the Creative Commons Attribution-NonCommercial-NoDerivs 4.0 International License (CC BY-NC-ND 4.0), which permits the noncommercial replication and distribution of the article with the strict proviso that no changes or edits are made and the original work is properly cited (including links to both the formal publication through the relevant DOI and the license). See: https://creativecommons.org/licenses/by-nc-nd/4.0/.

\section{References}

1. Lee ATJ, Thway K, Huang PH, et al. Clinical and Molecular Spectrum of Liposarcoma. J Clin Oncol 2018;36:151-9.

2. Matthyssens LE, Creytens D, Ceelen WP. Retroperitoneal liposarcoma: current insights in diagnosis and treatment. Front Surg 2015;2:4.

3. Gronchi A, Collini P, Miceli R, et al. Myogenic differentiation and histologic grading are major prognostic determinants in retroperitoneal liposarcoma. Am J Surg Pathol 2015;39:383-93.

4. Messiou C, Morosi C. Imaging in retroperitoneal soft tissue sarcoma. J Surg Oncol 2018;117:25-32.

5. Shaaban AM, Rezvani M, Tubay M, et al. Fat-containing Retroperitoneal Lesions: Imaging Characteristics, Localization, and Differential Diagnosis. Radiographics 2016;36:710-34.

6. Bhosale P, Wang J, Varma D, et al. Can Abdominal Computed Tomography Imaging Help Accurately Identify a Dedifferentiated Component in a Well-Differentiated
Liposarcoma? J Comput Assist Tomogr 2016;40:872-9.

7. Dodd LG. Update on liposarcoma: a review for cytopathologists. Diagn Cytopathol 2012;40:1122-31.

8. Tseng WW, Madewell JE, Wei W, et al. Locoregional disease patterns in well-differentiated and dedifferentiated retroperitoneal liposarcoma: implications for the extent of resection? Ann Surg Oncol 2014;21:2136-43.

9. Gronchi A, Strauss DC, Miceli R, et al. Variability in Patterns of Recurrence After Resection of Primary Retroperitoneal Sarcoma (RPS): A Report on 1007 Patients From the Multi-institutional Collaborative RPS Working Group. Ann Surg 2016;263:1002-9.

10. Haas RLM, Bonvalot S, Miceli R, et al. Radiotherapy for retroperitoneal liposarcoma: A report from the Transatlantic Retroperitoneal Sarcoma Working Group. Cancer 2019;125:1290-300.

11. McGovern Y, Zhou CD, Jones RL. Systemic Therapy in Metastatic or Unresectable Well-Differentiated/ Dedifferentiated Liposarcoma. Front Oncol 2017;7:292.

12. Zaidi MY, Canter R, Cardona K. Post-operative surveillance in retroperitoneal soft tissue sarcoma: The importance of tumor histology in guiding strategy. J Surg Oncol 2018;117:99-104.

13. Ikoma N, Roland CL, Torres KE, et al. Salvage Surgery for Recurrent Retroperitoneal Well-Differentiated Liposarcoma: Early Reoperation may not Provide Benefit. Ann Surg Oncol 2018;25:2193-200.

14. Mansfield SA, Pollock RE, Grignol VP. Surgery for Abdominal Well-Differentiated Liposarcoma. Curr Treat Options Oncol 2018;19:1.

15. Jung E, Fiore M, Gronchi A, et al. Second Primary Malignancies in Patients with Well-differentiated/ Dedifferentiated Liposarcoma. Anticancer Res 2018;38:3535-42.

16. Gronchi A, Lo Vullo S, Fiore M, et al. Aggressive surgical policies in a retrospectively reviewed single-institution case series of retroperitoneal soft tissue sarcoma patients. J Clin Oncol 2009;27:24-30.

17. Bonvalot S, Rivoire M, Castaing $M$, et al. Primary retroperitoneal sarcomas: a multivariate analysis of surgical factors associated with local control. J Clin Oncol 2009;27:31-7.

18. Binh MB, Sastre-Garau X, Guillou L, et al. MDM2 and CDK4 immunostainings are useful adjuncts in diagnosing well-differentiated and dedifferentiated liposarcoma subtypes: a comparative analysis of 559 soft tissue neoplasms with genetic data. Am J Surg Pathol 2005;29:1340-7. 
19. Clay MR, Martinez AP, Weiss SW, et al. MDM2 and CDK4 Immunohistochemistry: Should It Be Used in Problematic Differentiated Lipomatous Tumors?: A New Perspective. Am J Surg Pathol 2016;40:1647-52.

20. Tseng WW, Tsao-Wei DD, Callegaro D, et al. Pancreaticoduodenectomy in the surgical management of primary retroperitoneal sarcoma. Eur J Surg Oncol 2018;44:810-5.

21. MacNeill AJ, Gronchi A, Miceli R, et al. Postoperative Morbidity After Radical Resection of Primary Retroperitoneal Sarcoma: A Report From the Transatlantic RPS Working Group. Ann Surg 2018;267:959-64.

22. Quinones-Baldrich W, Alktaifi A, Eilber F, et al. Inferior vena cava resection and reconstruction for retroperitoneal tumor excision. J Vasc Surg 2012;55:1386-93; discussion 1393.

23. Ferraris M, Callegaro D, Barretta F, et al. Outcome of iliocaval resection and reconstruction for retroperitoneal sarcoma. J Vasc Surg Venous Lymphat Disord 2019;7:547-56.

24. Jiang H, Wang YX, Li B, et al. Surgical management of leiomyosarcoma of the inferior vena cava. Vascular 2015;23:329-32.

25. Miao C, Xiao M, Li T, et al. Difference in left renal

Cite this article as: Tseng WW, Chen J, Patel D, Miao C, Ching A, Yang S, Feng Y, Matcuk G, Lara K, Hu J, Ragab O, Ho J, Rao X, Hanif S, Luo C. Multidisciplinary sarcoma tumor board: retroperitoneal liposarcoma. Chin Clin Oncol 2020;9(2):20. doi: 10.21037/cco.2020.02.08 vein pressure: an indicator for free of reconstruction after ligation in retroperitoneal tumor patients. Sci Rep 2015;5:18126.

26. Italiano A, Toulmonde M, Cioffi A, et al. Advanced welldifferentiated/dedifferentiated liposarcomas: role of chemotherapy and survival. Ann Oncol 2012;23:1601-7.

27. Livingston JA, Bugano D, Barbo A, et al. Role of chemotherapy in dedifferentiated liposarcoma of the retroperitoneum: defining the benefit and challenges of the standard. Sci Rep 2017;7:11836.

28. Tawbi HA, Burgess M, Bolejack V, et al. Pembrolizumab in advanced soft-tissue sarcoma and bone sarcoma (SARC028): a multicentre, two-cohort, single-arm, openlabel, phase 2 trial. Lancet Oncol 2017;18:1493-501.

29. Dickson MA, Schwartz GK, Keohan ML, et al. Progression-Free Survival Among Patients With WellDifferentiated or Dedifferentiated Liposarcoma Treated With CDK4 Inhibitor Palbociclib: A Phase 2 Clinical Trial. JAMA Oncol 2016;2:937-40.

30. Yihebali Chi et al. Anlotinib for metastasis soft tissue sarcoma: A randomized, double-blind, placebocontrolled and multi-centered clinical trial. J Clin Oncol 2018;36:abstr 11503. 